\section{CONTRIBUIÇĀO AO ESTUDO DA ORIGEM E RAMIFICAÇĀO DAS ARTÉRIAS MESENTÉRICAS CRANIAL E CAUDAL, EM FETOS DE BOVINOS AZEBUADOS*}

\section{FREDERICO OZANAM CARNEIRO E SILVA \\ Professor Assistente \\ Universidade Federal de Uberlândia-MG}

JOSÉ PEDUTI NETO

Professor Adjunto

Faculdade de Medicina Veterinária e

Zootecnia da USP

PEDRO PRIMO BOMBONATO

Professor Assistente Doutor

Faculdade de Medicina Veterinária e

Zootecnia da USP

SILVA, F.O.C.; PEDUTI NETO, J.; BOMBONATO, P.P. Contribuição ao estudo da origem e ramificaçăo das artérias mesentéricas cranial e caudal, em fetos de bovinos azebuados. Rev.Fac. Med.vet.Zootec.Univ.S.Paulo, 22(1):31-36, 1985.

RESUMO: Foram estudados 30 fetos de bovinos azebuados (15 machos e 15 fêmeas), injetados com soluçào de Neoprene latex 450 e fixados em solução de formol a $10,0 \%$, tendo-se verificado que a artéija mesentérica cranial origina-se da artéria aorta abdominal, caudalmente à artéria celíaca ou em tronco comum com esta, emitindo a ses peculiares. Para a artéria mesentérica caudal notou-se sua origem sempre da artéria aorta abdominal, próximo aos seus ramos terminais, emitindo comumente a artéria cólica esquerda cranialmente e continuando-se como artéria retal cranial.

UNITERMOS: Anatomia, bovinos ${ }^{+}$; Artérias mesentéricas ${ }^{+}$; Bovinos azebuados

\footnotetext{
"Tese de mestrado apresentada à Faculdade de Medicina Veterinária e Zootecnia da USP.
}

\section{INTRODUÇÃO E LITERATURA}

Procurando melhor conhecer as caracteristicas morfológicas dos Bos indicus que se apresentam como de significativo valor na exploração zootécnica em -várias regiōes do Brasil, buscamos agora examinar novos e, eventualmente, particulares aspectos relativos à distribuiçáo dos ramos viscerais da artéria aorta, visando despertar a atenção dos pesquisadores no sentido de obter-se subsídios que permitam uma abordagem mais acertada em direção a uma exploração mais racional da criação destes animais, como também no aprimoramento deste grupo racial.

Assim, nesta oportunidade, propusemo-nos desenvolver trabalho, concemente à origem e distribuição dos ramos das artérias mesentéricas cranial e caudal, em bovinos azebuados, levando-se em conta o fato da quase totalidade da literatura consultada não trazer dados preciosos.

A escassez de literatura especializada fica patente, quando observamos que apenas PEDUTI NETO \& PRADA ${ }^{18}$; ARAÚJO ${ }^{1}$ e GODINHO et alii 11 apresentam dados específicos sobre os bovinos de origem indiana, já que os tratados clássicos nāo definem a origem dos animais por eles estudados, mas devido a suas procedencias, provavelmente o fizeram em bovinos de raças européias.

De sorte que PEDUTI NETO \& PRADA 18 j estudando em 46 fetos de bovinos, observaram que as Aa. celíaca e mesentérica cranial originavam por tronco comum em $6,5 \%$ das peças, ou seja, em 3 casos. Já, ARAƯJo ${ }^{1}$ notou que em 30 fetos examinados a origem acima citada ocorria em $13,3 \%$ dos casos, ou seja, em 4 casos. GODINHO et aliili, sem se prenderem a informaçōes numéricas, afirmam que a $\mathrm{A}$. mesenté̉ica cranial é a resultante caudal da bifurcação do tronco celíaco-mesentérico, que $e$ frequente nos ruminantes.

Autores como ARAÚJO ${ }^{1}$, BOSSI $^{2}$; BOURDELLE ${ }^{3}$; BRUNI \& ZIMMERL ${ }^{4}$; CHAUVEAU et alii ${ }^{5}$; DOBBERSTEIN \& HOFFMANN 6 ; ELLENBERGER \& BAUM $^{7}$; FAVILLI $^{8}$, FRANCK ${ }^{9}$, GETTY ${ }^{10}$, GODINHO et aliili. GONZÄLEZ Y GARCIA \& GONZÁLEZ ÄLVAREZ ${ }^{12}$, HABEL ${ }^{13}$, KOCH $^{14}$, MARTIN ${ }^{15}$, MARTIN \& SCHAUDER ${ }^{16}$, MONTANÉ et aliil ${ }^{17}$, PEDUTI NETO \& PRADA $^{18}$, SCHWARZE \& SCHRODER ${ }^{19}$; SISSON \& GROSSMAN ${ }^{20}$; ZIETZSCHMANN et alii2 ${ }^{21}$, ZIMMERL et alii22, descrevem, por vezes com outra nomenclatura, a origem da A. mesentérica cranial como sendo da A. aorta mas $\mathrm{KOCH}^{14}$, SCHWARZE \& SCHRరDER ${ }^{19}$ e GETTY ${ }^{10}$ relatam a possibilidade da referida artéria originar-se em tronco com a A. celíaca. Somente SISSON \&GROSSMAN 20 assinalam a eventualidade da $A$. mesentérica cranial ser ramo da A. celíaca.

No tocante aos ramos da A.mesentérica cranial, us autores já citados identificam os ramos pancreáticos, a A. pancreáticoduoduenal caudal, a A. cólica média, a A. ilioceco- 
cólica, o ramo colateral, as Aa. jejunais e as Aa. ileais, atendo-se apenas na descrição dos seus comportamentos e territórios de nutrição. No pertinente a A. cólica média, cabe ressaltar que DOBBERSTEIN \& HOFFMANN ${ }^{6}$ e $\mathrm{KOCH}^{14}$ citam a anastomose desta artéria com A. cólica esquerda.

Ao tratarem da origem e dos ramos da A. mesentérica caudal, os autores são concordes em afirmar ser tal vaso originado da face ventral da $\mathrm{A}$. aorta, próximo à sua terminação em correspondência d̀ última vértebra lombar, sendo sempre ímpar e emitindo, após um curto trajeto, as Aa. cólica esquerda e retal cranial, utilizando-se, entretanto, de nomenclaturas próprias e considerando apenas diferenças de calibres e áreas de vascularização.

\section{MATERIAL E MÉTODO}

Para esta pesquisa, valemo-nos de 30 fetos de bovinos azebuados, 15 machos (Obs. 16 a 30) e 15 fémeas (Obs. 1 a 15) , considerados como pontos de reparo, cranialmente a crista nucal e caudalmente a articulação entre as duas primeiras vértebras coccígeas, material este obtido em frigoríficos do Municipio de Uberlândia, Estado de Minas Gerais.

Assim, o preparo dos animais contava, inicialmente, com a abertura da cavidade torácica pelo lado esquerdo, ao nivel do nono espaço intercostal, seguindo-se individualização da A. aorta, pars thoracica - na qual, mediante cânula de calibre compativel ao diâmetro do vaso, procedíamos a injeção do referido sistema com solução de Neoprene latex " 450 ", corado em verde com pigmento específico*. As peças assim preparadas e após fixaçāo em solução aquosa de formol a $10,0 \%$, foram dissecadas, tendo-se executada previamente a abertura da cavidade abdominal, mediante incisóes, uma de sentido dorso ventral que tangenciava a borda caudal do arco costal e outra sobre a linha alba que se prolongava até à extremidade cranial da sínfise pübica.

Finalmente, os dados compilados através de desenhos foram estatisticamente tratados com auxilio do teste $\mathrm{X}^{2}$ $\operatorname{com} \alpha=5,0 \%$.

\section{RESULTADOS}

\section{A. Mesentérica Cranial}

Originando-se da face ventral da A. aorta, porção abdominal, a A. mesentérica cranial nasce separadamente da A. celíaca, 29 vezes $(96,66 \% \pm 3,3)$ ou em tronco comum com esta, 1 vez $(3,33 \% \pm 3,3)$ para, a seguir, fornecer ramos em número variável, a saber:

- Ramos adrenais esquerdos presentes 27 vezes $(90,0 \% \pm$

- 5) com número de vasos variando de um a quatro, sendo a maior frequência de dois -12 vezes $(36,66)$, um 19 vezes $(33,3 \%)$ e seis -3 vezes $(10,0 \%)$, quatro -2 vezes $(6,66 \%)$.

* Du Pont do Brasil S.A.

Rev.Fac.Med.vet Zootec.Univ.S.Paulo, 22(1):31-36, 1985.
- Ramos adrenais direitos, verificados 5 vezes $(16,66 \% \pm$ $6,8)$, cedendo uma -4 vezes $(13,33 \%)$ ou duas -1 vez $(3,33 \%)$ artérias.

- Ramos diafragmáticos, presentes 8 vezes $(26,66 \% \pm 8,1)$ contribuindo com ramos em número de um -6 vezes $(20,0 \%)$ ou dois -2 vezes $(6,66 \%)$. Cabe-nos considerar. a existência de um tronco comum entre um ramo diafragmático e um adrenal, mais especificamente com o ramo adrenal esquerdo, 1 vez $(3,33 \% \pm 3,3)$ e com o ramo adrenal direito, 1 vez $(3,33 \% \pm 3,3)$. A este nivel, identificamos um ramo anastomótico entre a $\mathrm{A}$. mesentérica cranial e a A. celíaca por 1 vez $(3,33 \% \pm 3,3)$.

- Ramos pancreáticos, notados em todas as preparaçōes, com maior frequência de un ramo - 20 vezes $(66,66 \%)$ e dois -10 vezes $(33,33 \%)$.

- A. pancreaticoduodenal cranial, presente em todas as peças, sendo que surpreendemos sua origem em tronco comum com uma A. jejuna'., 1 vez $(3,33 \% \pm 3,3)$ e ainda em tronco comum com as Aa. jejunal e cólica média por mais 1 vez $(3,33 \% \pm 3,3)$. Observamos que a referida artéria termina anastomosando-se com A. pancreaticoduodenal cranial.

- A. cólica média, identificada em todas as observações, acrescentando-se que é vista nascendo em tronco comum com uma A. jejunal, 2 vezes $(6,66 \%)$ e com ramos cólicos de A. mesentérica cranial, 1 vez $(3,33 \% \pm 3,3)$ e ain. da com as Aa. pancreaticoduodenal cranial e jejunal - 1 vez $(3,3 \% \pm 3,3)$. A artéria termina por anastomosar-se com a A. cólica esquerda, em todas as peças, e com ramos cólicos da A. mesentérica cranial, 1 vez $(3,33 \% \pm$ 3,3).

- A. ileocecocólica está presente 29 vezes $(96,66 \% \pm 3,3)$ cabendo salientar que no caso restante, 1 vez $(3,33 \% \pm$ $3,3)$ a citada artéria toma origem do ramo colateral. Ainda, em todos os casos cede ramos cólicos, cecal, ileal, bem como as Aa. ileocecal e ileal, sendo que anastomoses entre os referidos ramos e destes com os ramos terminais da A. mesentérica cranial (Aa ileais) e ramo colateral foram observados em todas as peças.

- Ramo colateral, identificado 29 vezes $(96,66 \% \pm 3,3)$ destacando-se as anastomoses que trava com as Aa. mesentérica cranial, jejunais e ileocecocólica.

- Aa. jejunais, presentes em todas as preparaçōes e notadas em número de três a sete, até a emissão do ramo colate$\mathrm{ral}$, com maior frequência de cinco -9 vezes $(30,0 \%)$, quatro -6 vezes $(30,0 \%)$, seis -6 vezes $(20,0 \%)$, sete -6 vezes $(20,0 \%)$ e três -2 vezes $(6,66 \%)$ artérias. A. nastomoses entre estes ramos foram sempre identificadas.

- Ramo cólico, observado por 6 vezes $(20,0 \% \pm 7,3)$ sempre em número de um.

- A. ileal, identificada em todas as peças, destacando que sempre termina por anastomosar-se com os ramos germinais da A. ileocecocólica. 


\section{A. Mesentérica Caudal}

Em todas as oportunidades é ímpar e vista a nascer da face ventral da A. aorta abdominal, próxima à sua terminação. Após um curto trajeto craniocaudal emite os seguintes ramos:

- A. cólica esquerda dirige-se cranialmente, envia uma série de ramos cólicos e termina anastomosando-se com a $\mathbf{A}$. cólica média, proveniente da A. mesentérica cranial. Esta presente em todas as nossas observaçס̄es.

- A. retal cranial, constitui-se na continuação direta da A. mesentérica caudal, dirige-se caudalmente e envia, ainda, série de ramos cólicos, sendo tal descrição sempre notada em nossas peças.

- Ramos cólicos, identificados na totalidade das peças, sendo seu número variado.

- Aa. ováricas, supreendemo-las emergindo diretamente da A. mesentérica caudal por $1 \mathrm{vez}(3,33 \% \pm 3,3)$.

Finalmente, analisando estatisticamente a participação dos ramos das Aa. mesentéricas cranial e caudal, não evidenciamos diferenças significantes no comportamento destes, em relação ao sexo.

\section{COMENTÁRIOS}

Sobre a origem da A. mesentérica cranial

Podemos afirmar que a maioria dos tratadistas ao abordarem o assunto fazem mençōes genéricas e por vezes imprecisas, relatando que a $\mathrm{A}$. mesentérica cranial ou $\mathrm{A}$. grande mesentérica, como querem alguns, ou A. mesentérica anterior, como desejam outros, origina-se da A. aorta, caudalmente ou imediatamente atrás, próxima, e em tronco comum com a A. celiaca, podendo mesmo ser ramo do referido vaso. Nenhum deles relata a presença de um ramo anastomótico entre a A. mesentérica cranial e a A. celíaca, identificado por nós 1 vez $(3,33 \%)$.

Entretanto, podemos confrontar objetivamente nossos resultados com os descritos por PEDUTI NETO \& PRADA $^{18}$ e ARAÜJO 1 que,valendo-se respectivamente de 46 fetos e 30 fetos de bovinos de raças indianas indicam, por ordem, em três $(6,5 \%)$ e quatro $(13,3 \%)$ casos, a presença de tronco comum entre a $\mathrm{A}$. mesentérica cranial e a $\mathrm{A}$. zelíaca, fato por nós observado apenas em um $(3,33 \%)$ caso, já que a citação sobre tal fato, feita por $\mathrm{KOCH}^{14}$, SCHWARZE \& SCHROัDER ${ }^{19}$, GETTY $^{10}$ e SISSON \& GROSSMAN ${ }^{20}$,é genérica.

Sobre os ramos da A. mesentérica cranial

Uma vez que nenhum dos autores clássicos se detem 12 descriçāo da frequência dos ramos arteriais, notamos portanto, apenas diferenças na designação destes, uma vez fue nosso trabalho prendeu-se a observar tal fato, ficando prejudicada a comparação de dados da literatura e os por nós obtidos.

Salietamos, entretanto, que nenhum dos autores consultados se refere à possibilidade da A. ileocecocólica originar-se do ramo colateral, o que foi visto por nós 1 vez $(3,33 \%)$. No respeitante aos primeiros ramos da A. mesentérica cranial, por nós descritos, relembrando: ramo anastomótico entre as Aa. mesentérica cranial e celíaca, ramos adrenais direito e esquerdo, ramos para tecidos adjacentes e ramos diafragmáticos, não há nenhuma citação dos autores referendados, tanto no tocante à origem, quanto à ordem de emergênca e distribuição.

\section{Sobre a origem da $\mathbf{A}$. mesentérica caudal}

Em concordância com os relatos dos diferentes autores, observamos que a A. mesentérica caudal é sempre impar e origina-se da face ventral da A. aorta abdominal.

Sobre os ramos da A. mesentérica caudal

Cabe considerar, ainda, que os autores consultados o. ferecem relato semelhante aos resultados por nós notados, quando afirmam que, após um curto trajeto, a A. mesentérica caudal fornece a A. cólica esquerda e a A. retal cranial. Entretanto, nenhum dos citados tratadistas comenta a respeito dos ramos cólicos da $\mathrm{A}$. mesentérica caudal, notados em todas as nossas preparaçסes, bem como nāo fazem men( ção à Aa. ováricas direita e esquerda tomando origem da A. mesentérica caudal, fato identificado por nós em 1 caso $(3,33 \%)$. Apenas DOBBERSTEIN \& HOFFMAN 6 e $\mathrm{KOCH}^{14}$ relatam a anastomose entre a $A$. cólica esquerda $e$ a A. cólica média, observada em todas as nossas peças.

\section{CONCLUSOES}

A análise dos resultados agora obtidos permite-nos chegar às seguintes conclusoes:

1) a A. mesentérica cranial origina-se da face ventral da $\mathrm{A}$. aorta abdominal $(96,66 \%)$ ou em tronco comum com a A. celíaca $(3,33 \%)$;

2) a A. mesentérica cranial emite: ramos adrenais à esquerda $(90,0 \%)$ ramos adrenais a direita $(16,66 \%)$, ramos diafragmáticos $(26,66 \%)$; ramos aos tecidos adjacentes $(26,66 \%)$, ramos pancreáticos $(100,0 \%)$; A. pancreaticoduodenal caudal $(100,0 \%)$; A. cólica média $(100,0 \%)$; A. ileocecocólica $(96,66 \%)$; ramo colateral $(96,66 \%)$; Aa. jejunais $(100,0 \%)$; ramos cólicos $(20,0 \%)$ e A. ileais $(100,0 \%)$;

3) o ramo colateral pode emitir a A. ileocecocólica $(3,33 \%)$;

4) podem originar-se em tronco comum, os ramos diafragmáticos e ramos adrenais esquerdos $(3,33 \%)$; ramo diafragmático e ramo adrenal direito $\left(3,3, \sigma_{-}\right)$; ; . :creaticoduodenal caudal e jejunal $(3,33 \%)$; Aa. pancreaticoduodenal caudal, cólica média e jejunal $(3,33 \%)$; Aa. cólica média e jejunal (3,33\%); Aa. cólica média e jejunal 
$(100,0 \%)$ A. cólica média e ramos cólicos $(3,33 \%)$, respectivamente;

5) notamos a presença de ramo anastomótico entre as Aa. mesentérica cranial e celíaca $(3,33 \%)$;

6) a A. mesentérica caudal origina-se de face ventral da $\mathrm{A}$. aorta abdominal $(100,0 \%)$ e emite, sempre, as Aa. cólica esquerda, retal cranial e ramos cólicos;

7) a A. mesentérica caudal pode fornecer as Aa. ováricas direita e esquerda $(3,33 \%)$;

8) verificamos, em todas as, peças anastomoses entre as Aa. cólica esquerda e cólica média;

9) não foram assinaladas diferenças estatisticamente significantes, ao nível de 5,0\%, no comportamento dos ramos das Aa. mesentéricas cranial e caudal, no atinente ao sexo.

SILVA, F.O.C.; PEDUTI NETO, J.; BOMBONATO, P.P. Contribution to the study of the origin and ramification of the cranial and caudal mesenteric arteries in Zebu foetuses.
Rev.Fac.Med.vet.Zootec.Univ.S.Pa ulo22(1): 31-36, 1985.

SUMMARY: Reminding that the anatomical data in bovines usually regard european breeds (Bos taurus) we preposed to study now the pattern of the mesenteric arteries in zebu cattle (Bos indicus). Thus we have dissected 30 foetuses ( 15 males and 15 females) which were injected with Neoprene latex 450 and fixed in formol solution (10\%). The results obtained permit the following conclusions: 1 ) the cranial mesenteric artery arises from the abdominal aorta artery just after the colic artery or in common trunk with this and give up the following branches: adrenal artery, diaphagmatic artery, artery to the adjacent connective tissue, pancreatic artery, caudal pancreatic duodenal artery, median colic artery, which anastomoses with the left colic artery, that derives from the caudal mesenteric artery; other branches are the jejunal arteries which form anastomotic arcades, the collateral branch, ileocecocolic artery which give up colic branches and ileal and cecal arteries and then it finishs by anastomoses with these vessels and with the collateral branch and the jejunal arteries. The caudal mesenteric artery arises from the abdominal aorta artery near of its terminal branches; it gives up the left colic artery and following as cranial retal artery.

UNTTERMS: Anatomy of cattle ${ }^{+}$; Arteries $^{+}$; Mesenteric arteries; Azebuad bovine 


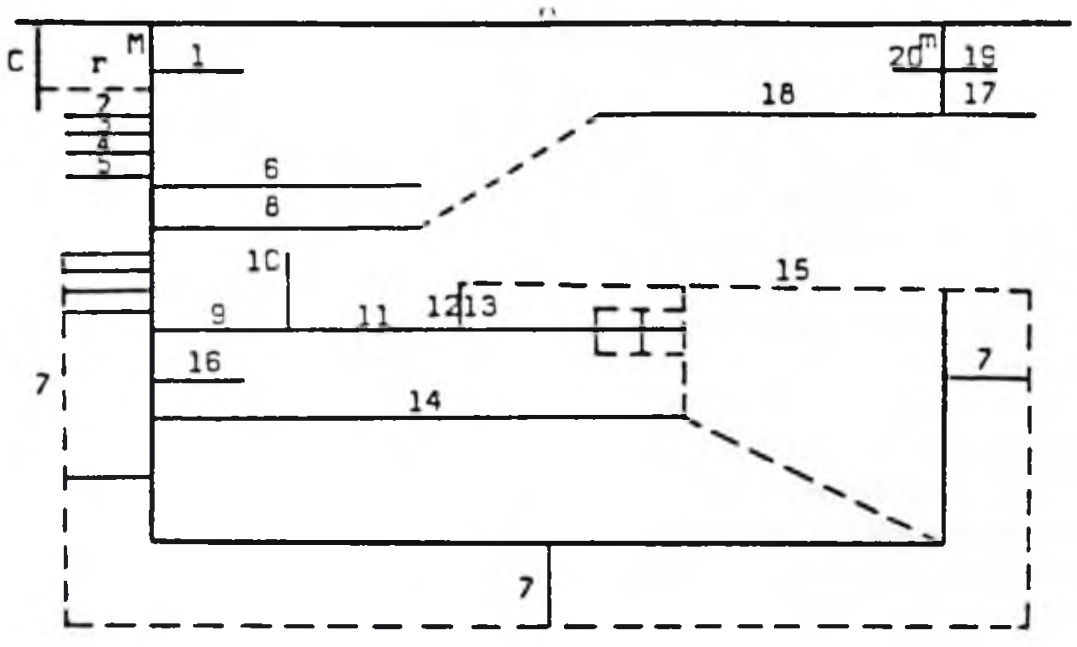

Esquema geral da origem dos ramos derivados das artérias mesentéricas cranial e caudal em fetos de bovinos azebuados.

\section{LEGENDA}

A. - Artéria aorta abdominal

T. - Tronco celíaco mesentérico

C. - Artéria celíaca

M. - Artéria mesentérica cranial

m. - Artéria mesentérica caudal

r. - Ramo comunicante entre E. e M.

1. - Ramo adrenal esquerdo

2. - Ramo adrenal direito

3. - Ramo para tecidos adjacentes

4. - Ramo diafragmático

5. - Ramo pancreático

6. - Artéria pancreaticoduodenal caudal

7. - Artérias jejunais

8. - Artéria cólica média

9. - Artéria ileocecocólica

10. - Ramo cólico

11. - Artéria ileocecal

12. - Artéria ileal

13. - Artéria cecal

14. - Ramo colateral

15. - Ramo ileal do $M$.

16. - Ramo cólico do M.

17. - Artéria retal cranial

18. - Artéria cólica esquerda

19. - Artéria ovárica direita

20. - Arté.: Jv'́. cuu uquerda

-.. Ramos anastomóticos

Rev.Fac.Med.vet.Zootec.Univ.S.Paulo, 22(1):31-36, 1985. 


\section{REFERENCLAS BIBLIOGRĀFICAS}

1 - ARAÚJO, J.C. Contribuição ao estudo dos ramos da artéria celíaca em fetos de bovinos azebuados. São Paulo, 1982. /Tese de mestrado - Faculdade de Medicina Veterinária e Zootecnia da Universidade de São Paulo/.

2- BOSSI, V. Angiologia. In: BOSSI, V.; CARADONNA, G.B.; SPAMPANI, G.; VARALDI, L. Trattato di anatomia veterinaria. Milano, Francesco Vallardi, s.d. v.2, p.205-6, 210.

3- BOURDELLE, E.; BRESSOU, C.; FLORENTIN, P. Technique de dissection des animaux domestiques. Paris, J.B. Baillière, 1947. p.192.

4- BRUNI, A.C. \& ZIMMERL, U. Anatomia degli animali domestici. 2. ed. Milano, Francesco Vallardi, 1947. v.2, p.350-1.

5- CHAUVEAU, A.; ARLOWG, S.; LESBRE, F.X. Précis d'anatomie comparé́ des animaux domestiques. Paris, J.B. Baillière, 1923. v.2, p. 366 .

6- DOBBERSTEIN, J. \& HOFFMANN, G. Lehrbuch der vergleichenden Anatomie der Haustiere. Leipzig, S. Hirzel, 1964. v.3, p.50.

7- ELLENBERGER, W. \& BAUM, H. Handbuch der vergleichenden Anatomie der Haustiere. 17. Auf. Berlin, Julius Springer, 1932. p.705-7.

8- FAVILLI, N. Nozioni comparata di anatomia e fisiologia degli animali rurali. Torino, Unione Tipografico-Editrice Toronese, 1931. p.403.

9- FRANCK, L. Handbuch der Anatomie der Haustiere. Stuttgart, Schickhardt \& Ebner, 1883. v.2, p.870-2.

10- GETTY, R. Anatomia dos animais domésticos. 5.ed. Rio de Janeiro, Interamericana, 1981, v.1, p.921-2. (Tradução de: Sisson and Grossman's The anatomy of domestic animals).

11 - GODINHO, H.P.; CA.RDOSO, F.M.; NASCIMENTO, J.F. Anatomia dos ruminantes domésticos. Be- lo Horizonte, 1981. p.372-4.

12- GONZÄLES Y GARCLA, J. \& GONZÁLES ÁlVAREZ, R. Anatomia comparada de los animales domesticos. 3.ed. Zaragoza, La Academica, 1929. p.634.

13- HABEL, R. Anatomia y manual de disseción de los ruminantes domesticos. Zaragoza, Acribia, 1968. p.47-8, 69 .

14- KOCH, T. Lehrbuch der Veterinăr-Anatomie. Jena, Gustav Fischer, 1965. v.3, p.120-4.

15- MARTIN, P. Lehrbuch der Anatomie der Haustiere. Stuttgart, Schickhardt \& Ebner, 1912. v.1 p.548.

16- MARTIN, P. \& SCHAUDER, W. Lehrbuch der Anatomie der Haustiere. Stuttgart, Schickhardt \& Ebner, 1935. v.3, p.227-9.

17- MONTANÉ, L. \& BOURDELLE, E. Anatomie régionale des animaux domestiques. Paris, J.B. Bailliere, 1917. v.2, p.275.

18- PEDUTI NETO, J. \& PRADA, I.L.S. Origem das ar. térias celíaca e mesentérica cranial, por tronco comum, em fetos de bovinos azebuados. Rev. Fac.Med.vet.Zootec.Univ.S.Paulo, $\quad 8: 399-402$ : 1970.

19- SCHWARZE, E. \&SCHRŐDER, L. Compêndio de anatomia veterinária. Zaragoza, Acribia, 1972. v.3, p.71.

20- SISSON, S. \& GROSSMAN, J.D. Anatomia de los animales domesticos. 4.ed. Barcelona, Salvat, 1959. p.705-6.

21- ZIETZSCHMANN, O.; ACKERKNECHT, GRAU, H. Ellenberger / Baum Handbuch der vergleichenden Anatomia der Haustiere. Berlin, Springer, 1943. p.680-1.

22- ZIMMERL, U.; BRUNI, A.C.; CARADONNA, G.B.; MANNU, ' ; .......USO, L. Trattato di anatomia veterinaria. Milano, Francesco Vallardi, 1930. v.2, p.1634, 167. 